\title{
Spontaneous angiogram-negative subarachnoid hemorrhage: a retrospective single center cohort study
}

\author{
Alexander Achrén $^{1}$ (D) $\cdot$ Rahul Raj $^{1} \cdot$ Jari Siironen ${ }^{1} \cdot$ Aki Laakso $^{1,2} \cdot$ Johan Marjamaa $^{1}$
}

Received: 23 April 2021 / Accepted: 12 November 2021 / Published online: 1 December 2021

(c) The Author(s) 2021

\begin{abstract}
Background Spontaneous angiogram-negative subarachnoid hemorrhage (SAH) is considered a benign illness with little of the aneurysmal SAH-related complications. We describe the clinical course, SAH-related complications, and outcome of patients with angiogram-negative $\mathrm{SAH}$.

Methods We retrospectively reviewed all adult patients admitted to a neurosurgical intensive care unit during 2004-2018 due to spontaneous angiogram-negative SAH. Our primary outcome was a dichotomized Glasgow Outcome Scale (GOS) at 3 months. We assessed factors that associated with outcome using multivariable logistic regression analysis.

Results Of the 108 patients included, $84 \%$ had a favorable outcome (GOS 4-5), and mortality was 5\% within 1 year. The median age was 58 years, $51 \%$ were female, and 93\% had a low-grade SAH (World Federation of Neurosurgical Societies grading I-III). The median number of angiograms performed per patient was two. Thirty percent of patients showed radiological signs of acute hydrocephalus, $28 \%$ were acutely treated with an external ventricular drain, $13 \%$ received active vasospasm treatment and $17 \%$ received a permanent shunt. In the multivariable logistic regression model, only acute hydrocephalus associated with unfavorable outcome (odds ratio $=4.05,95 \%$ confidence interval $=1.05-15.73$ ). Two patients had a new bleeding episode.

Conclusion SAH-related complications such as hydrocephalus and vasospasm are common after angiogram-negative SAH. Still, most patients had a favorable outcome. Only acute hydrocephalus was associated with unfavorable outcome. The high rate of SAH-related complications highlights the need for neurosurgical care in these patients.
\end{abstract}

Keywords Subarachnoid hemorrhage $\cdot$ Perimesencephalic subarachnoid hemorrhage $\cdot$ Intensive care unit $\cdot$ Spontaneous subarachnoid hemorrhage

\section{Introduction}

Spontaneous subarachnoid hemorrhage (SAH) causes 2-9\% of all strokes, but it is one of the most common causes of stroke-related deaths among the middle-aged [22, 25]. Ruptured intracranial aneurysms are the most common cause

This article is part of the Topical Collection on Vascular Neurosurgery - Other

Alexander Achrén

alexander.achren@helsinki.fi

1 Department of Neurosurgery, University of Helsinki and Helsinki University Hospital, Töölö Hospital, HUS, Topeliuksenkatu 5, PO. Box. 266, 00029 Helsinki, Finland

2 Division of Clinical Neuroscience, Department of Neurosurgery, University of Oulu, Oulu, Finland of spontaneous SAH (85\%). In approximately two-thirds of the remaining $(10 \%)$, the bleeding source cannot be identified; these are referred as spontaneous angiogram-negative $\mathrm{SAH}$; the residual (5\%) are due to various rare conditions consisting mainly of arteriovenous malformations [27]. These patients can be divided into two distinct categories based upon the distribution pattern of SAH. These include the perimesencephalic pattern $(\mathrm{PMH})$ with center of the blood pooled in cisterns around the midbrain or the diffuse pattern (nPMH) [23, 24]. Typically, both have a benign clinical course compared to aneurysmal SAH (aSAH) with fewer complications such as hydrocephalus, vasospasm, and delayed cerebral ischemia (DCI) [23, 27]. Furthermore, the prognosis of $\mathrm{PMH}$ and $\mathrm{nPMH}$ is considered good, with 83-100\% recovering with a favorable (4-5) Glasgow Outcome Score (GOS) compared to $59 \%$ of those with aSAH $[5,8,9,26]$. 
Few studies have looked at larger cohorts of patients with angiogram-negative SAH. A study by Little et al. [16] found those with nPMH to have worse outcome compared to $\mathrm{PMH}$, a $25 \%$ risk of angiographic vasospasm and a $6 \%$ risk for long-term hydrocephalus. Notably, is that $6-8 \%$ of patients have been reported to experience re-hemorrhage as a longterm complication after angiogram-negative $\mathrm{SAH}[3,23]$.

The aim with this retrospective cohort study is to describe the occurrence, clinical course, and outcome of patients with spontaneous angiogram-negative SAH treated in a large academic non-profit university hospital.

\section{Methods}

\section{Ethics}

The study was approved by the research committee of Helsinki University Hospital. Due to the retrospective study nature, no informed consent was obtained.

\section{Study setting}

We retrospectively reviewed all patients with angiogramnegative SAH admitted to the neurosurgical care unit (neuroICU) of Helsinki University Hospital from September 2004 to January 2018. Helsinki University Hospital is the largest university hospital in Finland, with a catchment area of approximately 2 million people (one third of the population in Finland). The treatment of SAH has been centralized to the neurosurgical department for decades.

\section{Patient population}

We screened all adult patients ( $\geq 18$ years) with an International Statistical Classification of Diseases and Health Problems, tenth revision (ICD-10) code of I60.7, I60.8, or I60.9 to the designated neuro-ICU. These patients' electronic healthcare records and radiological images were scrutinized. We considered only those with negative angiographic studies (including computed tomography angiogram, magnetic resonance angiogram, and digital subtraction angiogram). If the patient had an angiographic study, showing an intracranial aneurysm or other visible bleeding etiology the patient was not included. A minimum of one digital subtraction angiogram (DSA), computerized tomography angiogram (CTA), or magnetic resonance angiogram (MRA) examination was needed to rule out a ruptured aneurysm or other malformation. Patients with a history of trauma prior to the illness/ SAH were excluded, as were patients that were found to have vasculitis or reversible cerebral vasoconstriction syndrome.
At our institution, all conscious patients with SAH received per oral nimodipine and tranexamic acid (unconscious patients received intravenous nimodipine). Tranexamic acid was continued for 72 hours or until an aneurysmal bleeding source was excluded (after first or second angiography). After exclusion of aneurysmatic SAH, nimodipine was continued for 5 to 7 days.

\section{Data collection and definition of variables}

All data were collected through electronic healthcare records and radiological images. The data collected included patient characteristics, time of symptom onset, smoking habits, hypertension, antithrombotic medication, and number and type of performed angiograms together with length of hospital and ICU stay. Pupillary light reflexes, focal neurological symptoms, Glasgow Coma Scale (GCS) scores, the World Federation of Neurosurgical Societies (WFNS) grading scale, and Hunt and Hess $(\mathrm{H} \& \mathrm{H})$ scores were evaluated upon admission to the emergency department (ED) or preceding sedation and intubation. A WFNS score of 1-3 was considered low grade, and a score of 4-5 was considered high grade. Acute hydrocephalus was defined as an acute radiological enlargement of the brain ventricles, with or without treatment. The modified Fisher scale was based upon the admission imaging as were radiological appearance of a typical or atypical PMH.

Treatment-related variables such as hydrocephalus demanding external ventricular drainage (EVD), spinal drainage, or cerebrospinal fluid (CSF) shunt were collected.

Radiological vasospasm and delayed cerebral ischemia (DCI) were registered. DCI was defined as if a patient developed new neurological deficits not attributable to a previous focal lesion with or without radiological vasospasm. Actively treated vasospasm or DCI was registered. Generally, vasospasm treatment was initiated in case of DCI. Radiological vasospasm without clinical findings was not a treatment trigger. Yet, if a reliable neurological assessment was not possible, treatment was initiated in case of severe radiological vasospasm. Routine angiographic studies to diagnose or rule out radiological vasospasm were not performed.

\section{Outcomes}

Our primary outcome of interest was the Glasgow Outcome Score (GOS) 3 months after admission. The GOS was categorized into favorable (GOS 4-5) and unfavorable (GOS $\leq$ 3 ) outcome. Additionally, rebleeding rates (assessed August $1,2019)$ and 1-year case fatality are described as well. 


\section{Statistics}

Continuous variables were examined for distribution. Normally distributed data were analyzed using an independent $t$ test, whereas non-parametric data were analyzed with a non-parametric Mann-Whitney $U$ test. Categorical variables were compared between groups using a $\chi^{2}$ test (Fisher's exact test when needed). To assess the association between predictors and outcome, we first compared variables in univariate analysis. We considered $p$ values $<0.05$ as statistically significant. Significant not treatment-related variables that associated with outcome in the univariate analysis were included in a multivariable logistic regression model to find independent predictors associated with outcome. To avoid collinearity, we included WFNS grade instead of GCS score as WFNS grade is established in the SAH literature and allows for dichotomization into poor (WFNS IV to V) and good grade (WFNS I to III) [8]. We did not include variables from the univariate analysis with a high number of missing values. Age was included in all multivariable analyses. We separately analyzed patients with PMH and nPMH. SPSS Statistics version 25.0 for Windows (IBM Corp, Armonk, NY, USA) was used for all statistical analyses.

\section{Results}

Of 139 screened patients, a total of 130 patients met the inclusion criteria (Fig. 1). All SAH was diagnosed with a CT scan, except for two patients who had a normal CT scan and whose SAH was diagnosed with lumbar puncture. Of the 130 patients, 22 patients did not have any follow-up data. Thus, a total of 108 patients had compete follow-up data. There were no major differences in patient characteristics and treatment-related variables between patients with follow-up data and without followup data (Digital Supplemental 1).

Patient characteristics are shown in Table 1. The median age was 58 years, and approximately half were female. Fifteen percent were on some form of antithrombotic medication, and $36 \%$ had a previous diagnosis of hypertension. The majority of patients $(69 \%)$ had a GCS score of 15 on admission. Seven patients (7\%) were considered having high-grade SAH. Almost three out of four had a modified Fisher grade of 0 to 2, 54\% had intraventricular hemorrhage (IVH), 30\% had radiological and clinical signs of acute hydrocephalus, and $37 \%$ had a typical PMH distribution.

Of those with acute hydrocephalus, $56 \%$ needed acute CSF diversion and $44 \%$ had a ventriculoatrial or ventriculoperitoneal shunt.
Screened patients with

suspicion of non-aneurysmal

SAH during 2004-2018,

$n=139$

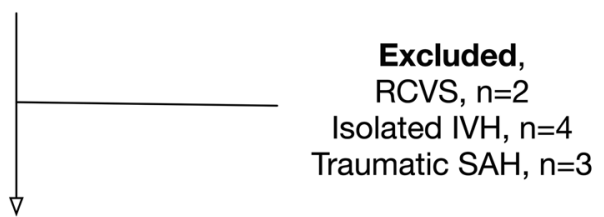

Non-aneurysmal SAH,

$n=130$

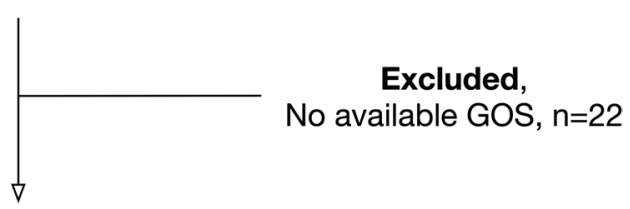

Study populations, $n=108$

Fig. 1. Flow chart showing study population

\section{Angiograms}

A total of 210 angiographic studies were performed on all patients. Of these $78 \%$ were CTA, 5\% MRA, and 17\% DSA. The median number of angiograms performed per patient was 2 (IQR 1-2). Sixty-three percent of all patients required a repeat angiogram, $24 \%$ of all patients underwent three or more angiograms, and $28 \%$ of all patients had at least one DSA performed.

\section{Treatment-related variables}

Thirty-nine patients (36\%) required some sort of CSF drainage. Of these, 30 patients had an EVD, 17 patients had a spinal drainage, and 18 patients received a ventriculoperitoneal or ventriculoatrial shunt (one patient could have one or several procedures). Median EVD treatment time was 8 days (IQR 5-14), and median spinal drainage time was 7 days (IQR 4-10). Median time from admission to shunting was 26 days (IQR 14-40). DCI was registered in $6 \%$ of patients. Isolated radiological vasospasm was registered in $16 \%$ of patients. Thirteen percent of patients received active vasospasm treatment.

\section{Patient outcomes}

The median time to GOS assessment was 82 days (IQR $52-105)$. The majority of patients $(84 \%)$ had a favorable functional outcome (GOS 4-5). Median length of stay in the hospital was 9 days (IQR 6-14), and median length of stay in the neuro-ICU was 2 days (IQR 1-7). Five patients (5\%) died within 1 year from admission. Only one patient died during the 
Table 1 Patient characteristics and treatment-related variables

\begin{tabular}{|c|c|}
\hline Variable & Patients $(n=108)$ \\
\hline Age, median (IQR) & $58(50-66)$ \\
\hline \multicolumn{2}{|l|}{ Sex } \\
\hline Female & $55(51 \%)$ \\
\hline Male & $53(49 \%)$ \\
\hline $\begin{array}{l}\text { Time from symptom onset to admission (days), } \\
\text { median (IQR) }\end{array}$ & $0(0-1)$ \\
\hline \multicolumn{2}{|l|}{ Smoking } \\
\hline Yes & $15(14 \%)$ \\
\hline No & $33(31 \%)$ \\
\hline Ex-smoker & $8(7 \%)$ \\
\hline Unknown & $52(48 \%)$ \\
\hline Antithrombotic medication & $16(15 \%)$ \\
\hline Anticoagulation & $6(6 \%)$ \\
\hline Antiplatelet & $8(7 \%)$ \\
\hline Both & $2(2 \%)$ \\
\hline No & $92(85 \%)$ \\
\hline Hypertension & $39(36 \%)$ \\
\hline \multicolumn{2}{|l|}{ GCS score } \\
\hline 15 & $75(69 \%)$ \\
\hline $13-14$ & $23(21 \%)$ \\
\hline $7-12$ & $7(7 \%)$ \\
\hline$<7$ & $3(3 \%)$ \\
\hline \multicolumn{2}{|l|}{ Pupillary light reactivity } \\
\hline Normal & $105(97 \%)$ \\
\hline Abnormal & $3(3 \%)$ \\
\hline Focal neurological symptom & $8(7 \%)$ \\
\hline \multicolumn{2}{|l|}{ WFNS grade } \\
\hline $1-3$ & $101(93 \%)$ \\
\hline $4-5$ & $7(7 \%)$ \\
\hline \multicolumn{2}{|l|}{ Radiological variables } \\
\hline \multicolumn{2}{|l|}{ Modified Fischer grade } \\
\hline 0 & $1(1 \%)$ \\
\hline 1 & $37(34 \%)$ \\
\hline 2 & $36(33 \%)$ \\
\hline 3 & $12(11 \%)$ \\
\hline 4 & $22(21 \%)$ \\
\hline IVH & $58(54 \%)$ \\
\hline Lateral ventricles & $30(28 \%)$ \\
\hline III ventricle & $23(21 \%)$ \\
\hline IV ventricle & $48(44 \%)$ \\
\hline Typical PMH distribution* & $40(37 \%)$ \\
\hline Acute hydrocephalus & $32(30 \%)$ \\
\hline \multicolumn{2}{|l|}{ Treatment-related variables } \\
\hline External ventricular drain & $30(28 \%)$ \\
\hline Time of drainage (days), median (IQR) $\dagger$ & $8(5-14)$ \\
\hline Spinal drainage & $17(16 \%)$ \\
\hline Time of drainage (days), median (IQR) $\neq$ & $7(4-10)$ \\
\hline CSF shunt & $18(17 \%)$ \\
\hline Days from admission to shunt, median (IQR) & $26(14-40)$ \\
\hline Radiological vasospasm & $17(16 \%)$ \\
\hline
\end{tabular}

Table 1 (continued)

\begin{tabular}{ll}
\hline Variable & Patients $(n=108)$ \\
\hline Clinical DCI & $6(6 \%)$ \\
Active DCI/vasospasm treatment & $14(13 \%)$ \\
ICU length of stay (days), median (IQR) & $2(1-7)$ \\
Hospital length of stay (days), median (IQR) & $9(6-14)$ \\
\hline
\end{tabular}

Abbreviations: $C S F$ cerebrospinal fluid; DCI Delayed Cerebral Ischemia; GCS Glasgow Coma Scale; $I C H$ intracerebral hemorrhage; $I C U$ intensive care unit; $I Q R$ interquartile range; $I V H$ intraventricular hemorrhage; $P M H$ perimesencephalic subarachnoid hemorrhage; WFNS World Federation of Neurosurgical Societies

*missing for 1 patient

$\dagger$ missing for 4 patients

$\ddagger$ missing for 3 patients

index hospitalization, 6 days after admission (massive SAH with hydrocephalus, IVH, and brain infarction). One of the deceased died due to diabetic ketoacidosis and infection during the convalescence 111 days later, the other patients died primarily from SAH related causes (Digital Supplemental 2).

\section{Re-bleedings}

Median rebleeding follow-up time was 7.6 years (IQR 3.3-11.4 years). One patient suffered a re-bleed 6 days after the initial SAH. For another patient, this hospitalization was a re-bleed episode from a previous spontaneous angiogramnegative SAH. These patients are more thoroughly described in the Digital Supplemental 2.

\section{Multivariable analysis}

Differences in patient characteristics between those with a favorable and unfavorable outcome are shown in Table 2. A total of 107 patients were included in the final multivariable logistic regression analysis (Table 3 ). Of the included variables, only acute hydrocephalus (odds ratio, OR 4.93; 95\% confidence interval, CI 1.14-21.41, $p=0.033$ ) showed an independent association with an unfavorable outcome.

\section{PMH versus $\mathrm{nPMH}$}

Differences between patients with favorable and unfavorable outcomes in the PMH and nPMH groups are shown in Table 4. In the PMH group, there were only two patients with unfavorable outcome, and, thus, a more detailed analysis regarding risk factors for unfavorable outcome was not possible.

For patients with nPMH, those with an unfavorable outcome had higher WFNS grade, higher modified Fisher grade, more frequent $\mathrm{IVH}$, and more frequent acute hydrocephalus. In a multivariable logistic regression model including these and age, only acute hydrocephalus 
Table 2 Patient characteristics and treatment-related variables according to functional outcome

\begin{tabular}{|c|c|c|c|}
\hline Variable & $\begin{array}{l}\text { Favorable outcome } \\
(N=91)\end{array}$ & $\begin{array}{l}\text { Unfavorable out- } \\
\text { come }(N=17)\end{array}$ & $p$ value \\
\hline Age, median (IQR) & $58(49-64)$ & $66(54-72)$ & 0.045 \\
\hline \multicolumn{4}{|l|}{ Sex } \\
\hline Female & $47(52 \%)$ & $8(47 \%)$ & \multirow[t]{2}{*}{0.795} \\
\hline Male & $44(48 \%)$ & $9(53 \%)$ & \\
\hline $\begin{array}{l}\text { Time from symptom onset to admission (days), } \\
\text { median (IQR) }\end{array}$ & $0(0-1)$ & $0(0-1)$ & 0.737 \\
\hline \multicolumn{4}{|l|}{ Smoking } \\
\hline Yes & $14(15 \%)$ & $1(6 \%)$ & \multirow[t]{4}{*}{0.014} \\
\hline No & $32(35 \%)$ & $1(6 \%)$ & \\
\hline Ex-smoker & $7(8 \%)$ & $1(6 \%)$ & \\
\hline Unknown & $38(42 \%)$ & $14(82 \%)$ & \\
\hline Antithrombotic medication & $10(11 \%)$ & $6(35 \%)$ & \multirow[t]{5}{*}{0.010} \\
\hline Anticoagulation & $2(2 \%)$ & $4(24 \%)$ & \\
\hline Antiplatelet & $6(7 \%)$ & $2(12 \%)$ & \\
\hline Both & $2(2 \%)$ & $0(0 \%)$ & \\
\hline No & $81(89 \%)$ & $11(65 \%)$ & \\
\hline Hypertension & $32(35 \%)$ & $7(41 \%)$ & 0.784 \\
\hline \multicolumn{4}{|l|}{ GCS score } \\
\hline 15 & $72(79 \%)$ & $3(18 \%)$ & \multirow[t]{4}{*}{$<0.001$} \\
\hline $13-14$ & $16(18 \%)$ & $7(41 \%)$ & \\
\hline $7-12$ & $2(2 \%)$ & $5(29 \%)$ & \\
\hline$<7$ & $1(1 \%)$ & $2(12 \%)$ & \\
\hline \multicolumn{4}{|l|}{ Pupillary light reactivity } \\
\hline Normal & $90(99 \%)$ & $15(88 \%)$ & \multirow[t]{2}{*}{0.064} \\
\hline Abnormal & $1(1 \%)$ & $2(12 \%)$ & \\
\hline Focal neurological symptom & $5(6 \%)$ & $3(18 \%)$ & 0.110 \\
\hline \multicolumn{4}{|l|}{ WFNS grade } \\
\hline $1-3$ & $88(97 \%)$ & $13(77 \%)$ & \multirow[t]{2}{*}{0.011} \\
\hline $4-5$ & $3(3 \%)$ & $4(23 \%)$ & \\
\hline \multicolumn{4}{|l|}{ Radiological variables } \\
\hline \multicolumn{4}{|l|}{ Modified Fisher grade } \\
\hline 0 & $1(1 \%)$ & $0(0 \%)$ & \multirow[t]{5}{*}{0.008} \\
\hline 1 & $34(37 \%)$ & $3(18 \%)$ & \\
\hline 2 & $31(34 \%)$ & $5(29 \%)$ & \\
\hline 3 & $12(13 \%)$ & $0(0 \%)$ & \\
\hline 4 & $13(14 \%)$ & $9(53 \%)$ & \\
\hline Modified Fisher grades 0-2 & $66(73 \%)$ & $8(47 \%)$ & \multirow[t]{2}{*}{0.049} \\
\hline Modified Fisher grades 3-4 & $25(28 \%)$ & $9(53 \%)$ & \\
\hline IVH & $44(49 \%)$ & $14(82 \%)$ & 0.015 \\
\hline Lateral ventricles & $17(19 \%)$ & $13(77 \%)$ & $<0.001$ \\
\hline III ventricle & $19(21 \%)$ & $4(24 \%)$ & 0.756 \\
\hline IV ventricle & $37(41 \%)$ & $11(65 \%)$ & 0.109 \\
\hline Typical PMH distribution* & $38(42 \%)$ & $2(13 \%)$ & 0.027 \\
\hline Acute hydrocephalus & $21(23 \%)$ & $11(65 \%)$ & 0.001 \\
\hline \multicolumn{4}{|l|}{ Treatment-related variables } \\
\hline External ventricular drain & $17(19 \%)$ & $13(77 \%)$ & $<0.001$ \\
\hline Time of drainage (days), median (IQR) $\dagger$ & $7(4-10)$ & $11(7-17)$ & 0.087 \\
\hline Spinal drainage & $13(14 \%)$ & $4(24 \%)$ & 0.456 \\
\hline Time of drainage (days), median (IQR) $\ddagger$ & $6(3-7)$ & $11(7-13)$ & 0.054 \\
\hline CSF shunt & $10(11 \%)$ & $8(47 \%)$ & 0.001 \\
\hline
\end{tabular}


Table 2 (continued)

\begin{tabular}{llll}
\hline Variable & $\begin{array}{l}\text { Favorable outcome } \\
(N=91)\end{array}$ & $\begin{array}{l}\text { Unfavorable out- } \\
\text { come }(N=17)\end{array}$ & $p$ value \\
\hline \multicolumn{1}{c}{ Days from admission to shunt, median (IQR) } & $25(4-52)$ & $28(19-33)$ & 0.696 \\
Radiological vasospasm & $13(14 \%)$ & $4(24 \%)$ & 0.466 \\
Clinical DCI & $5(6 \%)$ & $1(6 \%)$ & 1.000 \\
Active DCI/vasospasm treatment & $10(11 \%)$ & $4(24 \%)$ & 0.229 \\
ICU length of stay (days), median (IQR) & $2(1-4)$ & $10(3-19)$ & $<0.001$ \\
Hospital length of stay (days), median (IQR) & $9(6-13)$ & $14(5-32)$ & 0.088 \\
\hline
\end{tabular}

Favorable outcome is defined as a Glasgow Outcome Scale of 4-5, and unfavorable outcome is defined as a Glasgow Outcome Scale of 1-3.

Abbreviations: $C S F$ cerebrospinal fluid; $D C I$ delayed cerebral ischemia; $G C S$ Glasgow Coma Scale; $I C H$ intracerebral hemorrhage; ICU intensive care unit; IQR interquartile range; $I V H$ intraventricular hemorrhage; $P M H$ perimesencephalic subarachnoid hemorrhage; WFNS World Federation of Neurosurgical Societies

*missing for 1 patient

$\dagger$ missing for 4 patients

\$missing for 3 patients

remained associated with unfavorable outcome (OR 8.56, 95\% CI 1.59-46.23, Table 5).

Table 3 Multivariable logistic regression model showing predictors of poor functional outcome

\begin{tabular}{lll}
\hline Variable & OR $(95 \%$ CI $)$ & $p$ value \\
\hline $\begin{array}{l}\text { Age (cont.) } \\
\text { Antithrombotic medication }\end{array}$ & $1.05(0.99-1.12)$ & 0.106 \\
$\quad$ No & Ref & \\
$\quad$ Yes & $2.90(0.64-13.05)$ & 0.166 \\
WFNS SAH grade & & \\
1-3 & Ref & \\
4-5 & $2.93(0.42-20.66)$ & 0.281 \\
Modified Fisher grade & & \\
$0-2$ & Ref & 0.141 \\
$3-4$ & $3.16(0.68-14.57)$ & \\
IVH & & 0.173 \\
$\quad$ No & Ref & \\
Yes & $2.78(0.64-12.08)$ & \\
Acute hydrocephalus & & 0.033 \\
No & Ref & \\
Yes & $4.93(1.14-21.41)$ & 0.897 \\
Typical PMH & & \\
No & $1.14(0.16-8.23)$ & \\
Yes & Ref & \\
\hline
\end{tabular}

Favorable outcome is defined as a Glasgow Outcome Scale of 4-5, and unfavorable outcome is defined as a Glasgow Outcome Scale of $1-3$.

Abbreviations: $C I$ confidence interval; Cont. continuous; IVH intraventricular hemorrhage; $O R$ odds ratio; Ref reference; $S A H$ subarachnoid hemorrhage; $P M H$ perimesencephalic subarachnoid hemorrhage; WFNS World Federation of Neurosurgical Societies
Additionally, we compared patient characteristics between the PMH and nPMH group (Table 6). Patients with nPMH were more likely to have higher WFNS and modified Fisher grade, IVH in the lateral ventricles, acute hydrocephalus, permanent CSF shunt, and longer ICU and hospital stay.

\section{Discussion}

\section{Key findings}

We investigated the clinical course, SAH-related complications, and outcome of patients with angiogram-negative SAH over a 14-year period. We found that the vast majority of patients had a favorable neurological outcome. Still, $5 \%$ of patients died within one-year of admission. SAHrelated complications, such as hydrocephalus and vasospasm, were common but re-hemorrhage was rare.

We found greater age, smoking, antithrombotic medication, low GCS and WFNS upon administration, modified Fisher, presence of IVH, atypical PMH distribution, acute hydrocephalus, need of CSF diversion, and radiological vasospasm to associate with unfavorable outcome in univariate analysis. However, in multivariable logistic regression analysis, only acute hydrocephalus showed an independent association with unfavorable outcome.

\section{Comparison with previous studies}

Unfavorable outcome occurred in $16 \%$ of our patients, which is similar to previous studies $[9,14,16,20]$. Konczalla et al. found that $83 \%$ had an favorable neurological outcome, 
Table 4 Patient characteristics and treatment-related variables according to functional outcome for patients with perimesencephalic subarachnoid hemorrhage $(\mathrm{PMH})$ and diffuse pattern perimesencephalic subarachnoid hemorrhage (nPMH)

\begin{tabular}{|c|c|c|c|c|c|c|}
\hline \multirow[t]{2}{*}{ Variable } & \multicolumn{3}{|l|}{$\mathrm{PMH}(n=40)$} & \multicolumn{3}{|l|}{$\mathrm{nPMH}(n=67)$} \\
\hline & $\begin{array}{l}\text { Favorable out- } \\
\text { come }(N=38)\end{array}$ & $\begin{array}{l}\text { Unfavorable } \\
\text { outcome }(N=2)\end{array}$ & $p$ value & $\begin{array}{l}\text { Favorable out- } \\
\text { come }(N=53)\end{array}$ & $\begin{array}{l}\text { Unfavorable out- } \\
\text { come }(N=14)\end{array}$ & $p$ value \\
\hline Age, median (IQR) & $57(49-62)$ & $72(71-72)$ & 0.005 & $58(50-68)$ & $62(51-69)$ & 0.468 \\
\hline \multicolumn{7}{|l|}{ Sex } \\
\hline Female & $21(55 \%)$ & $1(50 \%)$ & 1.000 & $26(49 \%)$ & $7(50 \%)$ & 1.000 \\
\hline Male & $17(45 \%)$ & $1(50 \%)$ & & $27(51 \%)$ & $7(50 \%)$ & \\
\hline $\begin{array}{l}\text { Time from symptom onset to admission (days), } \\
\text { median (IQR) }\end{array}$ & $0(0-1)$ & $1(0-1)$ & 0.785 & $0(0-1)$ & $0(0-1)$ & 0.735 \\
\hline \multicolumn{7}{|l|}{ Smoking } \\
\hline Yes & $8(21 \%)$ & $0(0 \%)$ & 0.272 & $6(11 \%)$ & $1(7 \%)$ & 0.157 \\
\hline No & $15(40 \%)$ & $0(0 \%)$ & & $17(32 \%)$ & $1(7 \%)$ & \\
\hline Ex-smoker & $5(13 \%)$ & $1(50 \%)$ & & $2(4 \%)$ & $0(0 \%)$ & \\
\hline Unknown & $10(26 \%)$ & $1(50 \%)$ & & $28(53 \%)$ & $12(86 \%)$ & \\
\hline Antithrombotic medication & $35(92 \%)$ & $1(50 \%)$ & 0.192 & $7(13 \%)$ & $5(36 \%)$ & 0.109 \\
\hline Anticoagulation & $0(0 \%)$ & $0(0 \%)$ & & $2(4 \%)$ & $4(29 \%)$ & $\theta .039$ \\
\hline Antiplatelet & $3(8 \%)$ & $1(50 \%)$ & & $3(6 \%)$ & $1(7 \%)$ & \\
\hline Both & $0(0 \%)$ & $0(0 \%)$ & & $2(4 \%)$ & $0(0 \%)$ & \\
\hline No & $35(92 \%)$ & $1(50 \%)$ & & $46(87 \%)$ & $9(64 \%)$ & \\
\hline Hypertension & $12(32 \%)$ & $1(50 \%)$ & 1.000 & $20(38 \%)$ & $6(43 \%)$ & 0.765 \\
\hline \multicolumn{7}{|l|}{ GCS score } \\
\hline 15 & $30(79 \%)$ & $1(50 \%)$ & 0.086 & $42(79 \%)$ & $2(14 \%)$ & $<0.001$ \\
\hline $13-14$ & $8(21 \%)$ & $0(0 \%)$ & & $8(15 \%)$ & $6(43 \%)$ & \\
\hline $7-12$ & $0(0 \%)$ & $1(50 \%)$ & & $2(4 \%)$ & $4(29 \%)$ & \\
\hline$<7$ & $0(0 \%)$ & $0(0 \%)$ & & $1(2 \%)$ & $2(14 \%)$ & \\
\hline \multicolumn{7}{|l|}{ Pupillary light reactivity } \\
\hline Normal & $38(100 \%)$ & $2(100 \%)$ & & $52(98 \%)$ & $12(86 \%)$ & 0.108 \\
\hline Abnormal & $0(0 \%)$ & $0(0 \%)$ & & $1(2 \%)$ & $2(14 \%)$ & \\
\hline Focal neurological symptom & $2(5 \%)$ & $0(0 \%)$ & 1.000 & $3(6 \%)$ & $3(21 \%)$ & 0.100 \\
\hline \multicolumn{7}{|l|}{ WFNS grade } \\
\hline $1-3$ & $38(100 \%)$ & $2(100 \%)$ & & $50(94 \%)$ & $10(71 \%)$ & 0.030 \\
\hline $4-5$ & $0(0 \%)$ & $0(0 \%)$ & & $3(6 \%)$ & $4(29 \%)$ & \\
\hline \multicolumn{7}{|l|}{ Radiological variables } \\
\hline \multicolumn{7}{|l|}{ Modified Fisher grade } \\
\hline 0 & $0(0 \%)$ & $0(0 \%)$ & 1.000 & $1(2 \%)$ & $0(0 \%)$ & 0.047 \\
\hline 1 & $21(55 \%)$ & $1(50 \%)$ & & $13(25 \%)$ & $2(14 \%)$ & \\
\hline 2 & $17(45 \%)$ & $1(50 \%)$ & & $14(26 \%)$ & $3(21 \%)$ & \\
\hline 3 & $0(0 \%)$ & $0(0 \%)$ & & $12(23 \%)$ & $0(0 \%)$ & \\
\hline 4 & $0(0 \%)$ & $0(0 \%)$ & & $13(24 \%)$ & $9(64 \%)$ & \\
\hline Modified Fisher grades $0-2$ & $38(100 \%)$ & $2(100 \%)$ & & $28(53 \%)$ & $5(36 \%)$ & 0.369 \\
\hline Modified Fisher grades 3-4 & $0(0 \%)$ & $0(0 \%)$ & & $25(47 \%)$ & $9(64 \%)$ & \\
\hline IVH & $17(45 \%)$ & $1(50 \%)$ & 1.000 & $27(51 \%)$ & $12(86 \%)$ & 0.031 \\
\hline Lateral ventricles & $3(8 \%)$ & $1(50 \%)$ & 0.192 & $14(26 \%)$ & $11(79 \%)$ & $<0.001$ \\
\hline III ventricle & $6(16 \%)$ & $0(0 \%)$ & 1.000 & $13(25 \%)$ & $4(29 \%)$ & 0.740 \\
\hline IV ventricle & $15(40 \%)$ & $0(0 \%)$ & 0.519 & $22(42 \%)$ & $10(71 \%)$ & 0.071 \\
\hline Acute hydrocephalus & $5(13 \%)$ & $0(0 \%)$ & 1.000 & $16(30 \%)$ & $11(79 \%)$ & 0.002 \\
\hline \multicolumn{7}{|l|}{ Treatment-related variables } \\
\hline External ventricular drain & $4(11 \%)$ & $1(50 \%)$ & 0.237 & $13(25 \%)$ & $11(79 \%)$ & $<0.001$ \\
\hline Time of drainage (days), median (IQR) & $7(6-14)$ & NA & & $6(3-10)$ & $10(6-18)$ & 0.169 \\
\hline Spinal drainage & $4(11 \%)$ & $0(0 \%)$ & 1.000 & $9(17 \%)$ & $4(29 \%)$ & 0.447 \\
\hline
\end{tabular}


Table 4 (continued)

\begin{tabular}{|c|c|c|c|c|c|c|}
\hline \multirow[t]{2}{*}{ Variable } & \multicolumn{3}{|l|}{$\mathrm{PMH}(n=40)$} & \multicolumn{3}{|l|}{$\mathrm{nPMH}(n=67)$} \\
\hline & $\begin{array}{l}\text { Favorable out- } \\
\text { come }(N=38)\end{array}$ & $\begin{array}{l}\text { Unfavorable } \\
\text { outcome }(N=2)\end{array}$ & $p$ value & $\begin{array}{l}\text { Favorable out- } \\
\text { come }(N=53)\end{array}$ & $\begin{array}{l}\text { Unfavorable out- } \\
\text { come }(N=14)\end{array}$ & $p$ value \\
\hline Time of drainage $\dagger$ (days), median (IQR) & $6(6) \dagger$ & & & $4(2-8)$ & $11(7-13)$ & 0.042 \\
\hline CSF shunt & $1(3 \%)$ & $1(50 \%)$ & 0.099 & $9(17 \%)$ & $7(50 \%)$ & 0.029 \\
\hline Days from admission to shunt, median (IQR) & 2 & 34 & 1.000 & $26(10-55)$ & $26(18-30)$ & 1.000 \\
\hline Radiological vasospasm & $5(13 \%)$ & $1(50 \%)$ & 0.281 & $8(15 \%)$ & $3(21 \%)$ & 0.686 \\
\hline Clinical DCI & $2(5 \%)$ & $0(0 \%)$ & 1.000 & $3(6 \%)$ & $1(7 \%)$ & 1.000 \\
\hline Active DCI/vasospasm treatment & $3(8 \%)$ & $1(50 \%)$ & 0.192 & $7(13 \%)$ & $3(21 \%)$ & 0.425 \\
\hline ICU length of stay (days), median (IQR) & $1(1-2)$ & $10(3-19)$ & 0.974 & $2(1-6)$ & $12(6-20)$ & $<0.001$ \\
\hline Hospital length of stay (days), median (IQR) & $9(5-11)$ & $2(1-2)$ & 0.077 & $10(6-15)$ & $25(8-36)$ & 0.017 \\
\hline
\end{tabular}

Favorable outcome is defined as a Glasgow Outcome Scale of 4-5, and unfavorable outcome is defined as a Glasgow Outcome Scale of 1-3.

Abbreviations: $C S F$ cerebrospinal fluid; DCI delayed cerebral ischemia; GCS Glasgow Coma Scale; ICH intracerebral hemorrhage; ICU intensive care unit; IQR interquartile range; $I V H$ intraventricular hemorrhage; $P M H$ perimesencephalic subarachnoid hemorrhage; WFNS World Federation of Neurosurgical Societies

$\dagger$ missing for 3 patient

defined as modified Rankin Scale grading 0-2, in a cohort similar to our findings [14]. However, the all-cause mortality of $5 \%$ in our findings appears to be slightly higher compared to others. One study reported a mortality rate of $4 \%$ during a 45 month follow-up period [23], of which half died primarily from cancer, while another study reported a mortality rate as high as 10\%, 6 months after admission [14]. Our rebleeding

Table 5 Multivariable logistic regression model showing predictors of poor functional outcome in patients with diffuse pattern perimesencephalic subarachnoid hemorrhage

\begin{tabular}{lll}
\hline Variable & OR $(95 \%$ CI $)$ & $p$ value \\
\hline Age (cont.) & $1.03(0.97-1.10)$ & 0.338 \\
WFNS SAH grade & Ref & \\
$\quad 1-3$ & $2.74(0.41-18.47)$ & 0.299 \\
$\quad 4-5$ & & \\
Modified Fisher grade & Ref & \\
$\quad 0-2$ & $3.08(0.67-14.17)$ & 0.148 \\
$3-4$ & & \\
IVH & Ref & \\
$\quad$ No & $4.47(0.76-26.27)$ & 0.098 \\
$\quad$ Yes & & \\
Acute hydrocephalus & Ref & 0.013 \\
$\quad$ No & $8.56(1.59-46.23)$ & \\
$\quad$ Yes &
\end{tabular}

Favorable outcome is defined as a Glasgow Outcome Scale of 4-5, and unfavorable outcome is defined as a Glasgow Outcome Scale of $1-3$.

Abbreviations: $\mathrm{CI}$ confidence interval; Cont. continuous; $I V H$ intraventricular hemorrhage; $O R$ odds ratio; Ref reference; $S A H$ subarachnoid hemorrhage; $n P M H$ diffuse pattern perimesencephalic subarachnoid hemorrhage; WFNS World Federation of Neurosurgical Societies risk of $2 \%$ ( 2 patients) matches the previous numbers of $0-4 \%$ reported in previous studies after a follow-up ranging $0.8-90$ months $[3,13,18,20,23]$.

In the present study, only acute hydrocephalus was associated with unfavorable outcome. Acute hydrocephalus has also been described to associate with unfavorable outcome in other settings [14]. In comparison to previous studies, we did not find high grade SAH (defined as a WFNS of 4-5) to be associated with unfavorable outcome [14]. This is probably due to the low number of high grade SAH patients in our cohort (7 patients). Although it did not reach statistical significance, higher age seems to associate with a higher probability of unfavorable outcome.

Almost a third (30\%) of our patients displayed radiological signs of acute hydrocephalus. In comparison, approximately $40 \%$, of patients with aneurysmal SAH, show radiological signs of acute hydrocephalus on admission [6]. Our number seems rather high but is comparable to other series of angiogram-negative SAH patients (11-29\%) [9, 11, 13, $14,20]$. Reasons for the high occurrence of acute hydrocephalus are probably the high occurrence of IVH (54\%). Acute hydrocephalus is also associated with poorer outcome in aneurysmal SAH [5, 28]. Hydrocephalus associated poor outcome after SAH is probably related to a decrease in cerebral blood perfusion [26], stretching and damaging of the ventricle walls [19], and the corpus callosum [17].

Sixteen percent of our patients displayed signs of radiological vasospasm at some point during the hospitalization, while $13 \%$ received active vasospasm treatment. This number is lower than the occurrence of vasospasm in patients with aneurysmal SAH $(33 \%)[2,12,21]$. Also, previous studies with angiogram-negative SAH patients have showed similar rates of vasospasm (19\%) [15]. Still, whether the 
Table 6 Differences in patient characteristics and treatmentrelated variables for patients with perimesencephalic subarachnoid hemorrhage (PMH) and diffuse pattern Perimesencephalic Subarachnoid hemorrhage (nPMH)

\begin{tabular}{|c|c|c|c|}
\hline Variable & $\mathrm{PMH}(N=40)$ & $\mathrm{nPMH}(N=67)$ & $p$ value \\
\hline Age, median (IQR) & $58(49-63)$ & $59(50-67)$ & 0.230 \\
\hline \multicolumn{4}{|l|}{ Sex } \\
\hline Female & $22(55 \%)$ & $33(49 \%)$ & \multirow[t]{2}{*}{0.565} \\
\hline Male & $18(45 \%)$ & $34(51 \%)$ & \\
\hline $\begin{array}{l}\text { Time from symptom onset to admission (days), median } \\
\text { (IQR) }\end{array}$ & $0(0-1)$ & $0(0-1)$ & 0.823 \\
\hline \multicolumn{4}{|l|}{ Smoking } \\
\hline Yes & $8(20 \%)$ & $7(10 \%)$ & \multirow[t]{4}{*}{0.004} \\
\hline No & $15(38 \%)$ & $18(27 \%)$ & \\
\hline Ex-smoker & $6(15 \%)$ & $2(3 \%)$ & \\
\hline Unknown & $11(27 \%)$ & $40(60 \%)$ & \\
\hline Antithrombotic medication & $4(10 \%)$ & $12(18 \%)$ & \multirow[t]{5}{*}{0.402} \\
\hline Anticoagulation & $0(0 \%)$ & $6(9 \%)$ & \\
\hline Antiplatelet & $4(10 \%)$ & $4(6 \%)$ & \\
\hline Both & $0(0 \%)$ & $2(3 \%)$ & \\
\hline No & $36(90 \%)$ & $55(82 \%)$ & \\
\hline Hypertension & $13(33 \%)$ & $26(39 \%)$ & 0.512 \\
\hline \multicolumn{4}{|l|}{ GCS score } \\
\hline 15 & $31(78 \%)$ & $44(66 \%)$ & \multirow[t]{4}{*}{0.345} \\
\hline $13-14$ & $8(20 \%)$ & $14(21 \%)$ & \\
\hline $7-12$ & $1(2 \%)$ & $6(9 \%)$ & \\
\hline$<7$ & $0(0 \%)$ & $3(4 \%)$ & \\
\hline \multicolumn{4}{|l|}{ Pupillary light reactivity } \\
\hline Normal & $40(100 \%)$ & $64(96 \%)$ & \multirow[t]{2}{*}{0.291} \\
\hline Abnormal & $0(0 \%)$ & $3(4 \%)$ & \\
\hline Focal neurological symptom & $2(5 \%)$ & $6(9 \%)$ & 0.707 \\
\hline \multicolumn{4}{|l|}{ WFNS grade } \\
\hline $1-3$ & $40(100 \%)$ & $60(90 \%)$ & \multirow[t]{2}{*}{0.044} \\
\hline $4-5$ & $0(0 \%)$ & $7(10 \%)$ & \\
\hline \multicolumn{4}{|l|}{ Radiological variables } \\
\hline \multicolumn{4}{|l|}{ Modified Fisher grade } \\
\hline 0 & $0(0 \%)$ & $1(2 \%)$ & \multirow[t]{5}{*}{$<0.001$} \\
\hline 1 & $22(55 \%)$ & $15(22 \%)$ & \\
\hline 2 & $18(45 \%)$ & $17(25 \%)$ & \\
\hline 3 & $0(0 \%)$ & $12(18 \%)$ & \\
\hline 4 & $0(0 \%)$ & $22(33 \%)$ & \\
\hline Modified Fisher grades 0-2 & $40(100 \%)$ & $33(49 \%)$ & \multirow[t]{2}{*}{$<0.001$} \\
\hline Modified Fisher grades 3-4 & $0(0 \%)$ & $34(51 \%)$ & \\
\hline IVH & $18(45 \%)$ & $39(58 \%)$ & 0.185 \\
\hline Lateral ventricles & $4(10 \%)$ & $25(37 \%)$ & 0.002 \\
\hline III ventricle & $6(15 \%)$ & $17(25 \%)$ & 0.234 \\
\hline IV ventricle & $15(38 \%)$ & $32(48 \%)$ & 0.301 \\
\hline Acute hydrocephalus & $5(13 \%)$ & $27(40 \%)$ & 0.002 \\
\hline \multicolumn{4}{|l|}{ Treatment-related variables } \\
\hline External ventricular drain & $5(13 \%)$ & $24(36 \%)$ & 0.009 \\
\hline Time of drainage (days), median (IQR)* & $7(6-14)$ & $8(5-13)$ & 0.971 \\
\hline Spinal drainage & $4(10 \%)$ & $13(19 \%)$ & 0.277 \\
\hline Time of drainage (days), median (IQR) & $6(6)$ & $7(3-11)$ & 0.885 \\
\hline CSF shunt & $2(5 \%)$ & $16(24 \%)$ & 0.015 \\
\hline Days from admission to shunt, median (IQR) & $18(2-18)$ & $26(16-47)$ & 0.641 \\
\hline Radiological vasospasm & $6(15 \%)$ & $11(16 \%)$ & 1.000 \\
\hline Clinical DCI & $2(5 \%)$ & $4(6 \%)$ & 1.000 \\
\hline Active DCI/vasospasm treatment & $4(10 \%)$ & $10(15 \%)$ & 0.563 \\
\hline ICU length of stay (days), median (IQR) & $1(1-2)$ & $2(1-10)$ & 0.016 \\
\hline
\end{tabular}


Table 6 (continued)

\begin{tabular}{llll}
\hline Variable & PMH $(N=40)$ & $\mathrm{nPMH}(N=67)$ & $p$ value \\
\hline Hospital length of stay (days), median (IQR) & $8(4-11)$ & $10(6-17)$ & 0.044 \\
\hline
\end{tabular}

Favorable outcome is defined as a Glasgow Outcome Scale of 4-5, and unfavorable outcome is defined as a Glasgow Outcome Scale of 1-3.

Abbreviations: $C S F$ cerebrospinal fluid; $D C I$ delayed cerebral Ischemia; $G C S$ Glasgow Coma Scale; $I C H$ intracerebral hemorrhage; ICU intensive care unit; $I Q R$ interquartile range; $I V H$ intraventricular hemorrhage; $n P M H$ diffuse pattern perimesencephalic subarachnoid hemorrhage; $P M H$ perimesencephalic subarachnoid hemorrhage; WFNS World Federation of Neurosurgical Societies

*missing for 4 patients

†missing for 3 patients

pathophysiology leading to vasospasm and delayed cerebral ischemia after angiogram-negative $\mathrm{SAH}$ and aneurysmal SAH are similar remains unknown.

The etiology of spontaneous angiogram-negative SAH has yet to be established. It is thought to originate from a venous origin, given its amount of hemorrhage and benign clinical course [23]. A report by Hafez et al. found a venous saccular aneurysm in the lateral pontine vein, in a patient with PMH [7]. Another theory suggests a leakage from the ventriculostriate and thalamoperforating vessels [1]. Modern imaging methods enable more profound investigations of the arterial and venous systems that could help determine the origin for angiogram-negative SAH.

\section{Strengths and limitations}

There are some strengths that need to be highlighted. Because the care of SAH patients has been centralized to our department for decades, our cohort is comprehensive. Although there probably are angiogram-negative SAH patients never seeking hospital care, our study sample is representative of the situation in our catchment area during the study time. Further, healthcare records have stored electronically since the early 2000 s, making the data readily available and reliable.

However, some limitations should be mentioned. Our study is retrospective and our cohort stems from one center. Thus, generalizability of our results to other settings might be limited. All patients did not undergo DSA. At our institution, primary screening is done with CTA. Invasive DSA is mainly done to patients with a disproportionate amount of SAH or to patients in poor clinical condition. We had no structured follow-up of patients with angiogram-negative SAH, causing a $15 \%$ of lacking outcome data. Also, our cohort comes from a tertiary neuro-ICU. It is possible that some angiogram-negative SAH patients never seek medical care and thus, our, reported numbers (for example, $30 \%$ acute hydrocephalus) are overestimating the risk of SAH-related complications. Further, we assessed outcome according to GOS, which does not regard the patient's neuropsychological state or perceived quality of life. The neuropsychological component has been previously reported as an issue in patients with angiogram-negative $\mathrm{SAH}$, and it would be an implication for further research [4].

\section{Conclusions}

Although angiogram-negative SAH is considered a benign illness, we found rather high rates of SAH-related complications. Still, the vast majority did recover to a favorable outcome. Only acute hydrocephalus was associated with unfavorable outcome. The high rate of SAH-related complications highlights the need for neurosurgical care in these patients.

Supplementary Information The online version contains supplementary material available at https://doi.org/10.1007/s00701-021-05069-7.

Funding Open Access funding provided by University of Helsinki including Helsinki University Central Hospital. RR has received personal research grants from Finska Läkaresällskapet och Medicinska Understödsföreningen Liv \& Hälsa.

\section{Declarations}

Ethics approval The study was approved by the research committee of Helsinki University Hospital (\$3246), which waived the need for informed consent.

Conflict of interest The authors declare no competing interests.

Open Access This article is licensed under a Creative Commons Attribution 4.0 International License, which permits use, sharing, adaptation, distribution and reproduction in any medium or format, as long as you give appropriate credit to the original author(s) and the source, provide a link to the Creative Commons licence, and indicate if changes were made. The images or other third party material in this article are included in the article's Creative Commons licence, unless indicated otherwise in a credit line to the material. If material is not included in 
the article's Creative Commons licence and your intended use is not permitted by statutory regulation or exceeds the permitted use, you will need to obtain permission directly from the copyright holder. To view a copy of this licence, visit http://creativecommons.org/licenses/by/4.0/.

\section{References}

1. Alexander MSM, Dias PS, Uttley D (1986) Spontaneous subarachnoid hemorrhage and negative cerebral panangiography: Review of 140 cases. J Neurosurg 64(4):537-542

2. Allen GS, Ahn HS, Preziosi TJ et al (1983) Cerebral arterial spasm--a controlled trial of nimodipine in patients with subarachnoid hemorrhage. N Engl J Med 308(11):619-624

3. Boswell S, Thorell W, Gogela S, Lyden E, Surdell D (2013) Angiogram-negative subarachnoid hemorrhage: outcomes data and review of the literature. J Stroke Cerebrovasc Dis 22(6):750-757

4. Burke T, Hughes S, Carr A, Javadpour M, Pender N (2018) A systematic review of cognitive outcomes in angiographically negative subarachnoid haemorrhage. Neuropsychol Rev 28(4):453-469

5. Dupont S, Rabinstein AA (2013) Extent of acute hydrocephalus after subarachnoid hemorrhage as a risk factor for poor functional outcome. Neurol Res 35(2):107-110

6. Hadie A, Shen BV, Ville L et al (2016) Risk of shunting after aneurysmal subarachnoid hemorrhage. Stroke 47(10):2488-2496

7. Hafez A, Numminen $J$, Rahul R, Järveläinen $J$, Niemelä $M$ (2016) Perimesencephalic subarachnoid hemorrhage with a positive angiographic finding: case report and review of the literature. Acta Neurochir (Wien) 158(6):1045-1049

8. Jaja BNR, Saposnik G, Lingsma HF et al (2018) Development and validation of outcome prediction models for aneurysmal subarachnoid haemorrhage: the SAHIT multinational cohort study. BMJ 360:j5745

9. Jung JY, Kim YB, Lee JW, Huh SK, Lee KC (2006) Spontaneous subarachnoid haemorrhage with negative initial angiography: a review of 143 cases. J Clin Neurosci 13(10):1011-1017

10. Justiina H, Hanna L, Karri S, Riku K, Mika N, Juha H, Martin L (2015) Long-term excess mortality after aneurysmal subarachnoid hemorrhage. Stroke 46(7):1813-1818

11. Kang P, Raya A, Zipfel GJ, Dhar R (2016) Factors associated with acute and chronic hydrocephalus in nonaneurysmal subarachnoid hemorrhage. Neurocrit Care 24(1):104-109

12. Kassell NF, Torner JC, Haley EC, Jane JA, Adams HP, Kongable GL (1990) The international cooperative study on the timing of aneurysm surgery. Part 1: Overall management results. J Neurosurg 73(1): 18-36

13. Khan AA, Smith JDS, Kirkman MA, Robertson FJ, Wong K, Dott C, Grieve JP, Watkins LD, Kitchen ND (2013) Angiogram negative subarachnoid haemorrhage: outcomes and the role of repeat angiography. Clin Neurol Neurosurg 115(8):1470-1475

14. Konczalla J, Platz J, Schuss P, Vatter H, Seifert V, Güresir E (2014) Non-aneurysmal non-traumatic subarachnoid hemorrhage: patient characteristics, clinical outcome and prognostic factors based on a single-center experience in 125 patients. BMC Neurol 14(1):140

15. Lee SU, Hong EP, Kim BJ, Kim S-E, Jeon JP (2018) Delayed cerebral ischemia and vasospasm after spontaneous angiogramnegative subarachnoid hemorrhage: an updated meta-analysis. World Neurosurg 115:e558-e569
16. Little AS, Garrett M, Germain R, Farhataziz N, Albuquerque FC, McDougall CG, Zabramski JM, Nakaji P, Spetzler RF (2007) Evaluation of patients with spontaneous subarachnoid hemorrhage and negative angiography. Neurosurgery 61(6):1139-1151

17. Mataró M, Matarín M, Poca MA, Pueyo R, Sahuquillo J, Barrios M, Junqué C (2007) Functional and magnetic resonance imaging correlates of corpus callosum in normal pressure hydrocephalus before and after shunting. J Neurol Neurosurg Psychiatry 78(4):395-398

18. Mensing LA, Vergouwen Mervyn DI, Laban KG, Ruigrok YM, Velthuis BK, Ale A, Rinkel Gabriel JE (2018) Perimesencephalic Hemorrhage. Stroke 49(6):1363-1370

19. Miller JM, McAllister JP II (2007) Reduction of astrogliosis and microgliosis by cerebrospinal fluid shunting in experimental hydrocephalus. Cerebrospinal Fluid Res 4:5

20. Mohan M, Islim AI, Rasul FT et al (2019) Subarachnoid haemorrhage with negative initial neurovascular imaging: a systematic review and meta-analysis. Acta Neurochir (Wien) 161(10):2013-2026

21. Pickard JD, Murray GD, Illingworth R, Shaw MD, Teasdale GM, Foy PM, Humphrey PR, Lang DA, Nelson R, Richards P (1989) Effect of oral nimodipine on cerebral infarction and outcome after subarachnoid haemorrhage: British aneurysm nimodipine trial. BMJ 298(6674):636-642

22. Rautalin I, Kaprio J, Korja M (2020) Burden of aneurysmal subarachnoid haemorrhage deaths in middle-aged people is relatively high. J Neurol Neurosurg Psychiatry jnnp-2020-324706

23. Rinkel GJE, Wijdicks EFM, van Gijn J, Hasan D, Vermeulen M, Hageman LM, Kienstra GEM, Franke CL (1991) Outcome in patients with subarachnoid haemorrhage and negative angiography according to pattern of haemorrhage on computed tomography. The Lancet 338(8773):964-968

24. Rinkel GJ, Wijdicks EF, Vermeulen M, Ramos LM, Tanghe HL, Hasan D, Meiners LC, van Gijn J (1991) Nonaneurysmal perimesencephalic subarachnoid hemorrhage: CT and MR patterns that differ from aneurysmal rupture. AJNR Am J Neuroradiol 12(5):829-834

25. Sudlow CLM, Warlow CP (1997) Comparable studies of the incidence of stroke and its pathological types. Stroke 28(3):491-499

26. van Asch CJJ, van der Schaaf IC, Rinkel GJE (2010) Acute hydrocephalus and cerebral perfusion after aneurysmal subarachnoid hemorrhage. Am J Neuroradiol 31(1):67-70

27. van Gijn J, Rinkel GJE (2001) Subarachnoid haemorrhage: diagnosis, causes and management. Brain 124(2):249-278

28. Wang Y-M, Lin Y-J, Chuang M-J et al (2012) Predictors and outcomes of shunt-dependent hydrocephalus in patients with aneurysmal sub-arachnoid hemorrhage. BMC Surg 12:12

Comments The cause of spontaneous subarachnoid hemorrhage of both aneurysmal pattern and of nonaneurysmal perimesencephalic morphology remains obscure. In this paper, the authors review their experience with 108 such patients managed within a public health system. As previous studies have described, this kind of hemorrhage has an overall better prognosis than subarachnoid hemorrhage from an identified aneurysm. However, there is still considerable morbidity, especially in the perplexing group of patients who present with nonperimesencephalic hemoothage (in the authors' notation, nPMH). This group has a classic pattern of aneurysmal hemorrhage, but no aneurysm is ever found. In general, past experience has shown that for this group only very rarely is a cause identified and that rehemorrhage is rare. Their outcomes seem to track with the same kinds of factors that affect patients with aneurysmal patterns of hemorrhage: lower GCS, more severe WFNS or Fisher grade, and hydrocephalus. Accordingly, 
it seems appropriate that the nPMH patients' management should be very much like that of aneurysm patients, except that surgery and/ or endovascular treatment are not rendered at the beginning of their course of care. Nonetheless, they still warrant the full attention of neurosurgeons in that their critical care needs are still considerable. The patients with perimesencephalic hemorrhage $(\mathrm{PMH})$ still warrant aggressive care in terms of management of associated hydrocephalus --care which only neurosurgeons can provide-but fortunately their prognosis is otherwise very good in this series, which is consistent with the finding of others in the past. For both groups, the fact that the etiology still remains a mystery makes this an attractive area for ongoing study.
Peter Nakaji

Dara Sam Farhadi

Joseph Georges

Roland Jabre

Phoenix, Arizona, USA

Publisher's note Springer Nature remains neutral with regard to jurisdictional claims in published maps and institutional affiliations. 